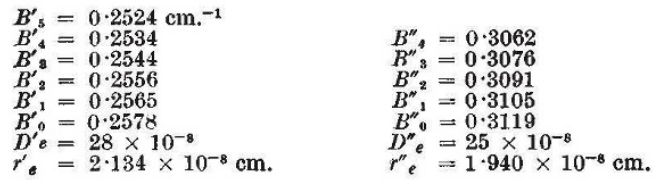

In the upper vibrational levels several perturbations have been found, which for $v^{\prime}=1,2$ and 3 are checked by observations on two bands for each $v^{\prime}$-level. This fact gives strong evidence for the correctness of our analysis. A more detailed paper will appear later.

Physics Department,

University, Stockholm.

AldBIN LAgERQVist ERLAND LIND

Physical Chemistry Laboratory,

University, Oxford.

July 17.

' Mecke, R., and Guillery, M., Phys. Z., 28, 514 (1928).

Mahanti, P. C., Proc. Phys. Soc., 46, 51 (1934).

'James, J. A., B.A. thesis, Oxford (1947).

\section{Diffraction Associated with Defocusing}

THE diffraction patterns near the focus in a plane perpendicular to the axis of a spherical wave front which passes through a circular aperture have been investigated by various authors ${ }^{1-7}$. The most complete treatment of this problem appears to be due to Lommel ${ }^{7}$, who derived expressions for the wavedisturbance in receiving planes other than the geometrical focal plane, and thus extended Airy's classical results ${ }^{2}$.

In some applications, particularly in those concerned with the performance of astronomical refracting telescopes, it is desirable not only to know the diffraction patterns but also to obtain a con. venient expression for the proportion of the total illumination falling outside a given circle with the axial point as centre. In the geometrical focal plane this is given by the well-known formula:

$$
J_{0}^{2}(v)+J_{1}^{2}(v),
$$

due to Rayleigh ${ }^{8}$.

Here $v=\frac{2 \pi R}{i f} r ; R$ denotes the aperture radius, $r$ the radius of the circle, $f$ the distance $C O$ between the plane of the aperture and the geometrical focus, and $\lambda$ is the wave-length.

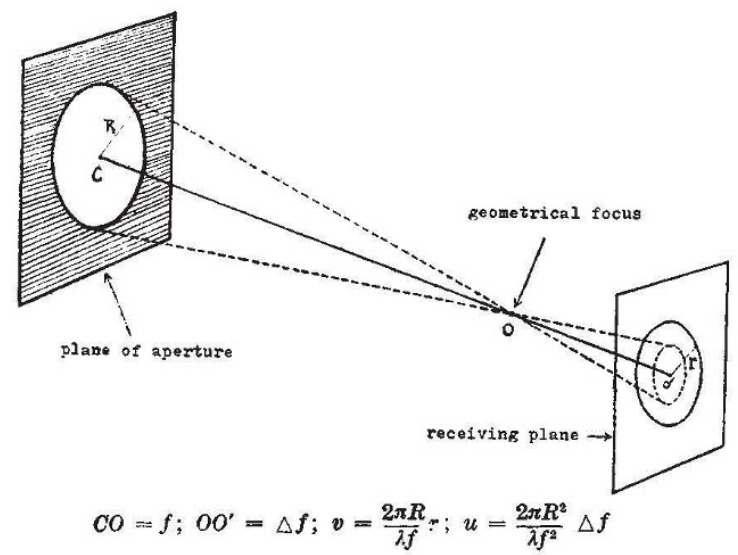

Starting from Lommel's formulæ, I have recently been able to generalize Rayleigh's result to the case when the receiving plane does not coincide with the geometrical focal plane, and obtain the following expressions for the fraction $L$ of the total illumination falling outside a circle of radius $r$ about the axial point, situated in a plane parallel to the aperture stop and distant $f+\Delta f$ from it :

$$
\begin{aligned}
& L=L(u, v)=1-\left(\frac{v}{u}\right)^{2}\left[1+\sum_{n=0}^{\infty} \frac{(-1)^{n}}{2 n+1}\left(\frac{v}{u}\right)^{2 n} P_{2 n}(v)\right] \\
& +\frac{4}{u}\left[Y_{1}(u, v) \cos \frac{1}{2}\left(u+\frac{v^{2}}{u}\right)+Y_{2}(u, v) \sin \frac{1}{2}\left(u+\frac{v^{2}}{u}\right)\right] \\
& \text { when }\left|\frac{v}{u}\right| \leqslant 1 \\
& =\sum_{n=0}^{\infty} \frac{(-1)^{n}}{2 n+1}\left(\frac{u}{v}\right)^{2 n} P_{2 n}(v) \quad \text { when }\left|\frac{v}{u}\right| \geqslant 1 \quad(2 b) \\
& \text { Here } u=\frac{2 \pi R^{2}}{f^{2}} \Delta f,
\end{aligned}
$$

so that $u= \pm v$ gives the edge of the geometrical shadow.

$Y_{1}$ and $Y_{2}$ are two of the functions

$$
Y_{n}(u, v)=\sum_{s=0}^{\infty}(-1)^{\delta}(n+2 s)\left(\frac{v}{u}\right)^{n+2 s} J_{n+2 s}(v)
$$

which have recently been introduced by Hopkins ${ }^{5}$, and the $P_{2 n}$ are certain polynomials involving Bessel functions, defined by the equation :

$$
\begin{aligned}
P(v)=\sum_{s=0}^{2 n}(-1)^{8} & \\
& \quad\left[J_{s}(v) J_{2 n-s}(v)+J_{s+1}(v) J_{2 n+1-s}(v)\right]
\end{aligned}
$$

When $u=0,(2 b)$ reduces to

$$
I(0, v)=P_{0}(v)=J_{0}^{2}(v)+J_{1}^{2}(v)
$$

in agreement with (1).

When $u= \pm v,(2 a)$ and $(2 b)$ reduce to

$$
L(v v)=J_{0}(v) \cos v+J_{1}(v) \sin v
$$

giving the fraction of illumination falling outside the purely geometrical confusion disk.

Equations ( $2 a)$ and $(2 b)$ make it possible to calculate the light scattered by diffraction from stellar images formed by refracting telescope objectives, taking into consideration the presence of the secondary spectrum. The results also have a bearing on the general question of 'distant diffracted light'. These questions, and the derivation of $(2 a)$ and $(2 b)$, will be discussed in more detail elsewhere.

\section{F. WoLF}

\section{Observatory, \\ Cambridge.}

June 18.

${ }^{1}$ Airy, G. B., Camb. Phil. Trans., 5, 283 (1835).

\& Buxton, A., Mon. Not. Roy. Astro. Soc., 81, 547 (1921).

"Conrady, A. E., Mon. Not. Roy. Astro. Soc., 79, 575 (1919 .

‘Epstein, L. I., J. Opt. Soc. Amer., 39, 226 (1949).

${ }^{5}$ Hopkins, H. H., Proc. Phys. Soc., 62, 22 (1949).

'Lansraux, G., Revue d'Optique, 26, 24 (1947).

'Lommel, W., Abh. Bayer. Akad. Wissensch., 15, 229 and 529 (1886). A more accessible, though condensed, account of Lommel's treatment is given in: Gray, A., Mathews, G. B., and MacRobert, T. M. "A Treatise on Bessel f'unctions" (second edit., Macmillan, London, 1931).

${ }^{8}$ Rayleigh, Phil. Mag., 11, 214 (1881). Also "Collected Papers", 1 , $513 ; 3,91$. 